\title{
NEUTRINO OSCILLATIONS AND LORENTZ VIOLATION
}

\author{
V. ALAN KOSTELECKÝ \\ Physics Department, Indiana University, Bloomington, IN 47405, U.S.A.
}

\begin{abstract}
Existing experimental data for neutrino oscillations are consistent with Lorentzviolating massless neutrinos. This talk summarizes some aspects of neutrino oscillations from the perspective of Lorentz and CPT violation in effective quantum field theory.
\end{abstract}

\section{Introduction}

Most data acquired to date by experiments in particle physics are successfully described by the minimal Standard Model (SM). One exception is the observation of neutrino oscillations, which are now well established and provide a compelling indication that the minimal SM requires modification. The inclusion of small masses in the neutrino sector of the SM suffices to describe the bulk of the experimental results, although a definitive understanding awaits the results of ongoing and future measurements. From a theoretical perspective, these modifications to the SM could arise as suppressed low-energy energy effects from an underlying unified theory at the Planck scale, $m_{P} \simeq 10^{19} \mathrm{GeV}$. This suggests the interesting possibility that neutrino oscillations might represent signals from the Planck scale.

This talk considers the description of neutrino oscillations in terms of a promising candidate Planck-scale signal, namely, violations of relativity arising through Lorentz and CPT breaking. ${ }^{1}$ The focus is on neutrino behavior from the perspective of Lorentz and CPT violation in effective quantum field theory. The general effective field theory describing Lorentz and CPT violation is called the Standard-Model Extension ${ }^{2,3}$ (SME) and was the basis for the original suggestion that Lorentz and CPT violation might occur in neutrinos. ${ }^{2}$ In the intervening years, several theoretical investigations of this possibility within the context of the SME have been performed, ${ }^{4,5,6,7,8,9,10,11}$ mostly for special models involving only a few coefficients for Lorentz and CPT violation. Here, a comprehensive theoretical study of Lorentz and CPT violation in neutrinos is outlined and a poten- 
tiallly realistic model is described. Details about the ideas discussed here can be found in the literature. ${ }^{9,10}$

\section{Theory}

The lagrangian of the SME consists of the SM and gravitational lagrangian supplemented by all possible coordinate-invariant terms constructed with SM and gravitational fields and violating Lorentz symmetry. ${ }^{3}$ The extra terms involve Lorentz-violating operators coupled to coefficients with Lorentz indices. Various origins have been suggested for these operators. A widely applicable and theoretically attractive source is physical spontaneous Lorentz violation, first suggested in gravitationally coupled field theories and string theory ${ }^{12}$ and then extended to incorporate CPT violation. ${ }^{13}$

Proposed sources also include noncommutative field theory, ${ }^{14}$ non-string approaches to quantum gravity, ${ }^{15}$ random dynamics, ${ }^{16}$ and multiverses. ${ }^{17}$ Measurements of SME coefficients for Lorentz violation have attained Planck-scale sensitivity in experiments with mesons, ${ }^{18,19,20}$ baryons, ${ }^{21,22,23}$ electrons, ${ }^{24,25}$ photons, ${ }^{26,27,28,29}$ and muons. ${ }^{30}$

This part of the talk considers the basic theory of neutrino behavior in the presence of Lorentz and CPT violation in Minkowski spacetime. The main focus is oscillation phenomena, ${ }^{31}$ but the formalism described can also be applied to other classes of experiments such as supernova studies, direct mass searches, and tests for neutrinoless double-beta decay.

\subsection{Effective hamiltonian}

Consider a general theory for $N$ neutrino species, incorporating all Majorana- and Dirac-type couplings of left- and right-handed neutrinos, both with and without Lorentz violation. Denote the neutrino fields by the Dirac spinors $\left\{\nu_{e}, \nu_{\mu}, \nu_{\tau}, \ldots\right\}$ and their charge conjugates $\left\{\nu_{e^{C}} \equiv \nu_{e}^{C}\right.$, $\left.\nu_{\mu^{C}} \equiv \nu_{\mu}^{C}, \nu_{\tau^{C}} \equiv \nu_{\tau}^{C}, \ldots\right\}$, where $\nu_{a}^{C} \equiv C \bar{\nu}_{a}^{T}$ as usual. Collect all fields and conjugates into a quantity $\nu_{A}$, where $A$ ranges over the $2 N$ possibilities $\left\{e, \mu, \tau, \ldots, e^{C}, \mu^{C}, \tau^{C}, \ldots\right\}$, so that charge conjugation becomes a linear transformation $\nu_{A}^{C}=\mathcal{C}_{A B} \nu_{B}$. In this notation, the dominant contributions to the general equations of motion for free propagation yield the first-order differential equation

$$
\left(i \Gamma_{A B}^{\nu} \partial_{\nu}-M_{A B}\right) \nu_{B}=0
$$

extending the usual equations of motion for Dirac and Majorana neutrinos. 
In Equation (1), $\Gamma_{A B}^{\mu}, M_{A B}$ are constant matrices in spinor space. They can be decomposed as

$$
\begin{aligned}
\Gamma_{A B}^{\nu} & \equiv \gamma^{\nu} \delta_{A B}+c_{A B}^{\mu \nu} \gamma_{\mu}+d_{A B}^{\mu \nu} \gamma_{5} \gamma_{\mu}+e_{A B}^{\nu}+i f_{A B}^{\nu} \gamma_{5}+\frac{1}{2} g_{A B}^{\lambda \mu \nu} \sigma_{\lambda \mu} \\
M_{A B} & \equiv m_{A B}+i m_{5 A B} \gamma_{5}+a_{A B}^{\mu} \gamma_{\mu}+b_{A B}^{\mu} \gamma_{5} \gamma_{\mu}+\frac{1}{2} H_{A B}^{\mu \nu} \sigma_{\mu \nu}
\end{aligned}
$$

Here, $m$ and $m_{5}$ are mass terms. All other coefficients control terms violating Lorentz symmetry, and the coefficients $a, b, e, f, g$ control CPT violation as well. The matrices $\Gamma_{A B}^{\mu}, M_{A B}$ must satisfy certain conditions following from hermiticity of the theory and from the interdependence of $\nu$ and $\nu^{C}$. For example, all the coefficients for Lorentz and CPT violation in Eq. (2) are hermitian in generation space.

Obtaining the hamiltonian associated with Eq. (1) requires handling the unconventional coefficient for the time derivative, which can be done following established procedures. ${ }^{32}$ For simplicity, consider here only the minimal case with $N=3$ and a standard seesaw mechanism ${ }^{33}$ suppressing the propagation of right-handed neutrinos, and restrict attention to leadingorder Lorentz and CPT violation. Amplitudes $b_{e, \mu, \tau}(t ; \vec{p})$ and $d_{e, \mu, \tau}(t ; \vec{p})$ can be defined that approximate active neutrinos and active antineutrinos of momentum $\vec{p}$. A somewhat lengthy calculation ${ }^{10}$ establishes that the time evolution of these amplitudes is

$$
\left(\begin{array}{c}
b_{a}(t ; \vec{p}) \\
d_{a}(t ; \vec{p})
\end{array}\right)=\exp \left(-i h_{\mathrm{eff}} t\right)_{a b}\left(\begin{array}{c}
b_{b}(0 ; \vec{p}) \\
d_{b}(0 ; \vec{p})
\end{array}\right)
$$

where $h_{\text {eff }}$ is the effective hamiltonian for flavor neutrino propagation, given at leading order by

$$
\begin{aligned}
& \left(h_{\mathrm{eff}}\right)_{a b}=|\vec{p}| \delta_{a b}\left(\begin{array}{ll}
1 & 0 \\
0 & 1
\end{array}\right)+\frac{1}{2|\vec{p}|}\left(\begin{array}{cc}
\left(\widetilde{m}^{2}\right)_{a b} & 0 \\
0 & \left(\widetilde{m}^{2}\right)_{a b}^{*}
\end{array}\right) \\
& +\frac{1}{|\vec{p}|}\left(\begin{array}{cc}
{\left[\left(a_{L}\right)^{\mu} p_{\mu}-\left(c_{L}\right)^{\mu \nu} p_{\mu} p_{\nu}\right]_{a b}} & -i \sqrt{2} p_{\mu}\left(\epsilon_{+}\right)_{\nu}\left[\left(g^{\mu \nu \sigma} p_{\sigma}-H^{\mu \nu}\right) \mathcal{C}\right]_{a b} \\
i \sqrt{2} p_{\mu}\left(\epsilon_{+}\right)_{\nu}^{*}\left[\left(g^{\mu \nu \sigma} p_{\sigma}+H^{\mu \nu}\right) \mathcal{C}\right]_{a b}^{*} & {\left[-\left(a_{L}\right)^{\mu} p_{\mu}-\left(c_{L}\right)^{\mu \nu} p_{\mu} p_{\nu}\right]_{a b}^{*}}
\end{array}\right) .
\end{aligned}
$$

In this equation, $\left(c_{L}\right)_{a b}^{\mu \nu} \equiv(c+d)_{a b}^{\mu \nu}$ and $\left(a_{L}\right)_{a b}^{\mu} \equiv(a+b)_{a b}^{\mu}, p_{\mu}=(|\vec{p}| ;-\vec{p})$ at leading order, and the complex vector $\left(\epsilon_{+}\right)_{\mu}$ can be chosen as $\left(\epsilon_{+}\right)^{\nu}=$ $\frac{1}{\sqrt{2}}\left(0 ; \hat{\epsilon}_{1}+i \hat{\epsilon}_{2}\right)$, where $\hat{\epsilon}_{1}, \hat{\epsilon}_{2}$ are real and $\left\{\vec{p} /|\vec{p}|, \hat{\epsilon}_{1}, \hat{\epsilon}_{2}\right\}$ form a right-handed orthonormal triad.

The first line of the above equation involves the diagonal kinetic term of the minimal SM and the usual Lorentz-conserving neutrino-mass term. In the second line, the coefficients $\left(a_{L}\right)_{a b}^{\mu},\left(c_{L}\right)_{a b}^{\mu \nu}$ determine the dominant Lorentz-violating effects in neutrino-neutrino mixing, while $\left(g^{\mu \nu \sigma} \mathcal{C}\right)_{a b}$, 
$\left(H^{\mu \nu} \mathcal{C}\right)_{a b}$ generate Lorentz-violating neutrino-antineutrino mixing. The former two types of coefficient preserve $\mathrm{SU}(3) \times \mathrm{SU}(2) \times \mathrm{U}(1)$ and appear in the minimal SME, while the latter two involve Majorana-type couplings breaking both $\mathrm{SU}(3) \times \mathrm{SU}(2) \times \mathrm{U}(1)$ and lepton number. Most of these coefficients lead to physical effects, but a few combinations are unphysical due to symmetries or to the existence of field redefinitions that can eliminate them. ${ }^{2,32,34,35}$ Note that the properties of the coefficients for Lorentz violation in Eq. (2) imply that the CPT-conjugate hamiltonian is obtained by changing the sign of the coefficients $a_{L}$ and $g$. Note also that none of the terms in Eq. (4) correspond to independent mass matrices for neutrinos and antineutrinos as recently proposed, ${ }^{36}$ a result consistent with Greenberg's formal impossibility proof. ${ }^{37}$

The effective hamiltonian (4) describes all contributions from operators of renormalizable dimension and therefore provides a definitive foundation for the treatment of Lorentz and CPT violation in neutrinos. Operators of nonrenormalizable mass dimension $n>4$ could also be significant, depending partly on the degree to which they are suppressed by powers of the Planck scale. ${ }^{19}$ In fact, at energies well beyond those relevant for current experiments, Lorentz-violating terms of nonrenormalizable dimension may be necessary for stability and causality. ${ }^{32}$ Some consequences of these more general operators are discussed in the literature..$^{9,10,38}$

The generality of the effective hamiltonian (4) implies that it must also contain the effects of matter interactions. Indeed, the effective lagrangian for neutrino propagation in normal matter is $\Delta \mathcal{L}_{\text {matter }}=$ $-\sqrt{2} G_{F} n_{e} \bar{\nu}_{e} \gamma^{0} P_{L} \nu_{e}+\left(G_{F} n_{n} / \sqrt{2}\right) \bar{\nu}_{a} \gamma^{0} P_{L} \nu_{a}$, which means that matter effects are incorporated by coefficient contributions of the form $\left(a_{L, \text { eff }}\right)_{e e}^{0}=$ $G_{F}\left(2 n_{e}-n_{n}\right) / \sqrt{2}$ and $\left(a_{L, \mathrm{eff}}\right)_{\mu \mu}^{0}=\left(a_{L, \mathrm{eff}}\right)_{\tau \tau}^{0}=-G_{F} n_{n} / \sqrt{2}$, where $n_{e}$ and $n_{n}$ are the number densities of electrons and neutrons.

\subsection{Neutrino mixing}

To extract the implications for neutrino mixing, it is useful to diagonalize the effective hamiltonian. This involves a $6 \times 6$ unitary matrix $U_{\text {eff }}$, $h_{\text {eff }}=U_{\text {eff }}^{\dagger} E_{\text {eff }} U_{\text {eff }}$, where $E_{\text {eff }}$ is a $6 \times 6$ diagonal matrix. There are therefore as many as six independent propagating states excluding sterile neutrinos, five possible eigenvalue differences, and hence five independent oscillation lengths in Lorentz-violating mixing. In contrast, the Lorentz-covariant case allows only three independent propagating states and two independent oscillation lengths. Note also that the five eigenvalue differences are not nor- 
mal mass differences since their energy dependences are unconventional.

Denote the six independent propagating states by the amplitudes $B_{J}(t ; \vec{p}), J=1, \ldots, 6$. Then, $B_{J}(t ; \vec{p})=\widetilde{U}_{J a} b_{a}(t ; \vec{p})+\bar{U}_{J a} d_{a}(t ; \vec{p})$, where $U_{\text {eff }}$ has been separated into $6 \times 3$ matrices $U_{\text {eff }}=(\widetilde{U}, \bar{U})$. The time evolution operator $S_{a b}(t)$ becomes

$$
S_{a b}(t)=\left(U_{\mathrm{eff}}^{\dagger} e^{-i E_{\mathrm{eff}} t} U_{\mathrm{eff}}\right)_{a b}=\left(\begin{array}{c}
S_{\nu_{a} \nu_{b}}(t) S_{\nu_{a} \bar{\nu}_{b}}(t) \\
S_{\bar{\nu}_{a} \nu_{b}}(t) S_{\bar{\nu}_{a} \bar{\nu}_{b}}(t)
\end{array}\right),
$$

where $E_{(J)}$ are the diagonal values of $E_{\text {eff }}$. This expression yields the oscillation probabilities at time $t$. Thus, the probability for a neutrino of type $b$ to oscillate into a neutrino of type $a$ is $P_{\nu_{b} \rightarrow \nu_{a}}(t)=\left|S_{\nu_{a} \nu_{b}}(t)\right|^{2}$, while that for a neutrino of type $b$ to oscillate into an antineutrino of type $a$ is $P_{\nu_{b} \rightarrow \bar{\nu}_{a}}(t)=\left|S_{\bar{\nu}_{a} \nu_{b}}(t)\right|^{2}$. Similarly, for antineutrinos we have $P_{\bar{\nu}_{b} \rightarrow \nu_{a}}(t)=$ $\left|S_{\nu_{a} \bar{\nu}_{b}}(t)\right|^{2}$ or $P_{\bar{\nu}_{b} \rightarrow \bar{\nu}_{a}}(t)=\left|S_{\bar{\nu}_{a} \bar{\nu}_{b}}(t)\right|^{2}$.

The CPT properties of the transition amplitudes are $S_{\nu_{a} \nu_{b}}(t) \longleftrightarrow$ $S_{\bar{\nu}_{a} \bar{\nu}_{b}}^{*}(-t)$ and $S_{\bar{\nu}_{a} \nu_{b}}(t) \longleftrightarrow-S_{\nu_{a} \bar{\nu}_{b}}^{*}(-t)$. If CPT is unbroken these relations become equalities, whereupon the first generates the standard result that CPT invariance implies $P_{\nu_{b} \rightarrow \nu_{a}}(t)=P_{\bar{\nu}_{a} \rightarrow \bar{\nu}_{b}}(t)$, while the second implies $P_{\nu_{b} \rightleftarrows \bar{\nu}_{a}}(t)=P_{\nu_{a} \rightleftarrows \bar{\nu}_{b}}(t)$. However, negation of terms in these results can fail. For example, CPT violation need not imply $P_{\nu_{b} \rightarrow \nu_{a}}(t) \neq P_{\bar{\nu}_{a} \rightarrow \bar{\nu}_{b}}(t)$.

For the above analysis of the effective hamiltonian and transition probabilities, the choice of observer reference frame is irrelevant because the physics is coordinate invariant and in particular is observer Lorentz invariant. ${ }^{2}$ However, since particle Lorentz symmetry is violated, ${ }^{2}$ it is advisable to adopt a standard frame to report experimental measurements of the coefficients for Lorentz violation. Conventionally, this is taken to be a Sun-centered celestial equatorial frame with coordinates $(T, X, Y, Z)$. The $Z$ direction is aligned with the Earth's rotational axis, and the $X$ direction points towards the vernal equinox. The coefficients for Lorentz violation in any inertial frame can be related to those in the standard Sun-centered frame by an appropriate observer Lorentz transformation. ${ }^{29}$ Since neutrino oscillations in the presence of Lorentz violation can exhibit orientationdependent effects, it is also convenient to define a standard parametrization in the Sun-centered frame for the direction of neutrino propagation $\hat{p}$ and for the $\hat{\epsilon}_{1}, \hat{\epsilon}_{2}$ vectors introduced above:

$$
\begin{aligned}
\hat{p} & =(\sin \Theta \cos \Phi, \sin \Theta \sin \Phi, \cos \Theta), \\
\hat{\epsilon}_{1} & =(\cos \Theta \cos \Phi, \cos \Theta \sin \Phi,-\sin \Theta), \\
\hat{\epsilon}_{2} & =(-\sin \Phi, \cos \Phi, 0) .
\end{aligned}
$$


Here, $\Theta$ and $\Phi$ are the celestial colatitude and longitude of propagation, respectively. This parametrization is assumed in what follows.

\section{Sensitivities}

No convincing experimental evidence for Lorentz violation presently exists. The size of theoretically predicted effects varies, but it is reasonable to expect $^{13}$ that observables for Lorentz and CPT violation are suppressed by some power of the dimensionless ratio $r=m / m_{P} \lesssim 10^{-17}$, where $m$ is the relevant low-energy scale and $m_{P}$ is the Planck mass. Note that the physics governing experiments on neutrino oscillations is controlled by the dimensionless ratio $r_{\nu}=\Delta m^{2} / E^{2}$, where $\Delta m^{2} \lesssim 10^{-20} \mathrm{GeV}$ and $10^{-4}$ $\mathrm{GeV}<E<10^{3} \mathrm{GeV}$. The ratios $r$ and $r_{\nu}$ are similar in range, suggesting that the natural scale for Lorentz violation could be comparable to the natural scale of neutrino oscillations.

Sensitivities to certain coefficients for Lorentz violation in the fermion and photon sectors have now reached parts in $10^{-30}$ or better. It might therefore seem plausible that theoretical considerations involving symmetries or radiative corrections could generate constraints on Lorentz violation in neutrinos from these impressive sensitivities in other sectors, but this idea largely fails in practice. At tree level, leading-order perturbative calculations involve only flavor-diagonal coefficients, and the Lorentz-violating charged-lepton and neutrino sectors are completely independent of these. Also, although radiative corrections can constrain a few neutrino effects under favorable circumstances, ${ }^{8}$ the properties of the electroweak sector typically ensure the independence of the charged- and neutral-lepton sectors at leading order in Lorentz violation even under radiative corrections. ${ }^{10}$

Various types of experiment can be sensitive to Lorentz- and CPTviolating effects, depending on the neutrino behavior studied. For example, the energy dependence of Lorentz-violating oscillations can be unconventional. Recall that the physically relevant dimensionless combination controlling neutrino oscillations induced by mass-squared differences $\Delta m^{2}$ is $\Delta m^{2} L / E$, where $L$ is the baseline distance and $E$ is the neutrino energy. In contrast, oscillations induced by Lorentz violation are controlled by the dimensionless combinations $a^{\mu} L, b^{\mu} L, H^{\mu \nu} L$ and $c^{\mu \nu} L E, d^{\mu \nu} L E$, $g^{\mu \nu \sigma} L E$, as readily seen from Eq. (4). In general, oscillations generated by a coefficient $\left(k_{d}\right)^{\lambda \ldots}$ for a Lorentz-violating operator of nonrenormalizable dimension $n=d+3$ are controlled by $\left(k_{d}\right)^{\lambda \cdots} L E^{d}$.

Another unconventional effect is direction-dependence of neutrino be- 
havior, which results from violation of rotation symmetry. ${ }^{10}$ This has implications both for comparisons between different experiments and for the analysis of experiments involving multiple sources. The orientation of the neutrino beam and the location of the source relative to the detector can affect neutrino oscillations. The daily rotation of the Earth induces apparent periodic changes of the coefficients for Lorentz violation in the laboratory that would appear as time variations in oscillation data at multiples of the sidereal frequency $\omega_{\oplus} \simeq 2 \pi /(23 \mathrm{~h} 56 \mathrm{~min})$. Also, observable annual variations in the solar-neutrino flux could arise from the change in the location of the detector as the Earth orbits the Sun.

Lorentz violation can also lead to novel resonance effects in neutrino oscillations. The usual MSW resonances ${ }^{39}$ occur when neutrino interactions with local matter become comparable to effects from mass, which alters the structure of the effective hamiltonian. Many other types of resonances can be induced by Lorentz violation, involving various combinations of coefficients for Lorentz violation, masses, and matter effects. For instance, resonances are possible with neither mass nor matter that are triggered by distinct coefficients for Lorentz violation. Known explicit examples include a two-generation vacuum resonance involving a single nonzero coefficient $\left(a_{L}\right)^{T}$ and a mass term, ${ }^{5}$ and a three-generation orientation-dependent vacuum resonance involving two coefficients $\left(a_{L}\right)^{Z}$ and $\left(c_{L}\right)^{T T}$ without mass. ${ }^{9}$

Certain experimental signals can be regarded as characteristic of Lorentz violation in neutrino oscillations. Six model-independent classes of signal exist that would offer evidence for Lorentz violation. ${ }^{10}$ Spectral anomalies can arise because each coefficient for Lorentz violation introduces unconventional energy dependence. These can generate complicated energy dependences in both oscillation lengths and mixing angles. $L-E$ conflicts represent any null or positive measurement in a region of $L-E$ space that conflicts with all mass-based scenarios. Of the six classes mentioned here, only this one presently has some positive evidence, the LSND anomaly. ${ }^{40}$ Periodic variations are induced by rotation-symmetry breaking and include both sidereal and annual variations. Sidereal variations can arise in experiments with Earth-based sources because the direction of neutrino propagation relative to the Sun-centered frame changes as the Earth rotates. Annual variations can arise in solar-neutrino experiments because the orientation of the detector relative to the Sun changes as the Earth orbits the Sun. Compass asymmetries also result from rotation-symmetry breaking, but the signals are independent of time. They would appear at the location of the detector as unexplained horizontal or vertical asymmetries. Neutrino-antineutrino 
mixing is a direct consequence of any model with nonzero coefficients of type $g$ or $H$. This class of signal involves lepton-number violation, and it includes in particular any appearance measurement that can be traced to $\nu \leftrightarrow \bar{\nu}$ oscillation. The final class of signal involves the classic CPT test of the relation $P_{\nu_{b} \rightarrow \nu_{a}} \neq P_{\bar{\nu}_{a} \rightarrow \bar{\nu}_{b}}$. This could also include violation of the condition $P_{\nu_{b} \rightleftarrows \bar{\nu}_{a}}(t)=P_{\nu_{a} \rightleftarrows \bar{\nu}_{b}}(t)$, which requires $\nu \leftrightarrow \bar{\nu}$ mixing.

Overall, the prospects are good for studies of Lorentz and CPT violation via neutrino oscillations. Consider an experiment with maximum $L$ coverage of $L_{\max }$ and maximum $E$ coverage of $E_{\max }$. A rough estimate of the sensitivity $\sigma$ to a coefficient for Lorentz violation of dimension $1-d$ can be taken $\operatorname{as}^{10} \sigma \approx-\log L_{\max }-d \log E_{\max }$. This can be used to show that all classes of experiment can attain Planck-scale reach for Lorentz and CPT violation, and the best may rival some of the most sensitive experiments in other sectors of the SME. Note also that non-oscillation experiments with neutrinos typically also have sensitivity to Lorentz violation, ${ }^{10}$ in particular via sidereal variations and compass asymmetries. This includes laboratory experiments such as direct mass searches or searches for neutrinoless doublebeta decay and also astrophysical observations of supernova neutrinos.

\section{Illustrative Models}

To gain insight into the variety of oscillation behavior exhibited by neutrinos in the presence of Lorentz violation, it is useful to consider special cases of the effective hamiltonian (4) involving only a few nonzero coefficients. In this part of the talk, some possible models of this type are first briefly discussed, and then a realistic example (the bicycle model) is considered.

\subsection{Basics}

One class of special models is obtained by requiring rotation symmetry. The resulting rotation-invariant or so-called 'fried-chicken' (FC) models are of definite interest for certain investigations because the rotation symmetry simplifies some calculations. It is worth noting, however, that these models are difficult to motivate theoretically as exact descriptions relevant to experimental studies. Thus, it might seem reasonable to require spherical symmetry in a special frame, perhaps the cosmic microwave background (CMB) frame. However, assuming rotation symmetry in the CMB frame means that the coefficients appearing in the effective hamiltonian (4) differ from those appearing in the standard Sun-centered frame or any other experimentally attainable frame. Converting between the CMB frame and 
the Sun-centered frame or other standard choice of experimentally attainable frame introduces direction dependence due to the solar-system motion relative to the CMB. This implies that the experimentally relevant hamiltonian also involves spatial components of the coefficients, suggesting that it cannot be an FC limit of the theory (4).

The general FC limit of the effective hamiltonian $h_{\mathrm{eff}}$ is straightforward to obtain. It contains four matrices, $\left(\widetilde{m}^{2}\right)_{a b},\left(a_{L}\right)_{a b}^{0},\left(c_{L}\right)_{a b}^{00},\left(c_{L}\right)_{a b}^{j k}=$ $\frac{1}{3}\left(c_{L}\right)_{a b}^{l l} \delta^{j k}$. However, only three of these are independent because the trace $\left(c_{L}\right)_{a b}^{00}-\left(c_{L}\right)_{a b}^{j j}$ is unobservable and can be set to zero. For definiteness, assume the rotation symmetry occurs in the Sun-centered $(T, X, Y, Z)$ frame. Then, the relevant part of the $6 \times 6$ effective hamiltonian reduces to the block-diagonal form

$$
\left(h_{\mathrm{eff}}\right)_{a b}^{\mathrm{FC}}=\left(\begin{array}{cc}
\left(\widetilde{m}^{2} / 2 E+\left(a_{L}\right)^{T}-\frac{4}{3}\left(c_{L}\right)^{T T} E\right)_{a b} & 0 \\
0 & \left(\widetilde{m}^{2} / 2 E-\left(a_{L}\right)^{T}-\frac{4}{3}\left(c_{L}\right)^{T T} E\right)_{a b}^{*}
\end{array}\right) .
$$

This equation determines the general FC model for three active neutrinos. Additional light or massless sterile neutrinos can be incorporated if needed.

Except for the original proposal for Lorentz violation in neutrinos ${ }^{2}$ and more recent papers, ${ }^{9,10,11}$ a sizable part of the literature ${ }^{4,5,6,7}$ concerns restricted special limits of the general FC model (7). In particular, most works have considered the two-generation special case in the limit of vanishing $\left(a_{L}\right)^{T}$ or $\left(c_{L}\right)^{T T}$. Even though the FC model (7) is somewhat restricted relative to the rich structure of the full effective hamiltonian (4), a more complete study would be of definite interest. A large class of oscillatory behaviors and their phenomenological implications in the FC limit remain unexplored to date.

In the more general context of the full effective hamiltonian (4), Lorentz violation implies directional dependence of oscillation physics through the breaking of rotation invariance. A variety of special cases involving directional dependence can be considered..$^{10}$ For example, one interesting limiting class of models with direction dependence consists of those with nonzero coefficients $g^{\mu \nu \sigma}$ and $H^{\mu \nu}$ only. These models necessarily involve $\nu \leftrightarrow \bar{\nu}$ mixing. In the general theory (4), nonzero $\nu \leftrightarrow \bar{\nu}$ mixing of this type can lead to five distinct oscillation lengths and corresponding complications.

A restriction of the effective hamiltonian (4) to the special case involving only the two-dimensional $\nu_{e}-\bar{\nu}_{e}$ subspace offers an interesting and readily workable limit with directional dependence. In this single-flavor limit of $\nu \leftrightarrow \bar{\nu}$ mixing, any coefficients $\left(\widetilde{m}^{2}\right)_{e e}$ and $\left(c_{L}\right)_{e e}$ can be ignored because they are real and lead to terms proportional to the identity that have no 
effect on oscillatory behavior. Furthermore, the coefficient $\left(H^{\mu \nu} \mathcal{C}\right)_{a b}$ is antisymmetric in generation space, so $\left(H^{\mu \nu} \mathcal{C}\right)_{e e}=H_{e e^{C}}^{\mu \nu}=0$. The most general single-flavor theory without mass differences is therefore given by a $2 \times 2$ effective hamiltonian containing only the coefficients $\left(a_{L}\right)_{e e}^{\mu}$ and $\left(g^{\mu \nu \sigma} \mathcal{C}\right)_{e e}=g_{e e^{C}}^{\mu \nu \sigma}$ for Lorentz violation. This model and any further restrictions necessarily violate CPT, since both $\left(a_{L}\right)_{e e}^{\mu}$ and $g_{e e C}^{\mu \nu \sigma}$ control CPT-odd terms in the hamiltonian. Note that the probabilities for this general singleflavor model have structure identical to that of the standard two-generation mixing case, $P_{\nu_{e} \leftrightarrow \bar{\nu}_{e}}=1-P_{\nu_{e} \rightarrow \nu_{e}}=1-P_{\bar{\nu}_{e} \rightarrow \bar{\nu}_{e}}=\sin ^{2} 2 \theta \sin ^{2} 2 \pi L / L_{0}$. However, the mixing angle and oscillation length depend on the 4-momentum in an unconventional and direction-dependent way.

The above discussion has focused on basic consequences of special models with only a small number of coefficients. However, even in apparently simple cases of these types, the neutrino behavior can be complicated and counterintuitive. An example of a counterintuitive phenomenon is given by the Lorentz-violating seesaw mechanism. ${ }^{10}$ This can arise when there are degeneracies in the low- or high-energy limit of $h_{\text {eff }}$. The different energy dependences among the coefficients for Lorentz violation in $h_{\mathrm{eff}}$ can then lead to the emergence of an oscillation length behaving like a mass-squared difference, despite the absence of mass-squared differences in the model.

To illustrate this, consider a $3 \times 3$ effective hamiltonian $h_{\text {eff }}$ parametrized in some basis as

$$
h_{\mathrm{eff}}=\left(\begin{array}{ccc}
2 h_{1} & h_{2} & h_{3} \\
h_{2}^{*} & 0 & 0 \\
h_{3}^{*} & 0 & 0
\end{array}\right),
$$

where irrelevant diagonal terms have been disregarded. For this class of toy models, the interesting eigenvalue difference is $\Delta=$ $\sqrt{\left(h_{1}\right)^{2}+\left|h_{2}\right|^{2}+\left|h_{3}\right|^{2}}-h_{1}$. If the combination coefficients for Lorentz violation is such that $h_{1} \gg \sqrt{\left|h_{2}\right|^{2}+\left|h_{3}\right|^{2}}$ holds at some energy scale, then the eigenvalue difference is approximately $\Delta \approx \frac{1}{2}\left(\left|h_{2}\right|^{2}+\left|h_{3}\right|^{2}\right) / h_{1}+\cdots$. For instance, suppose $h_{2}$ and $h_{3}$ originate from a dimension-one coefficient and hence are constant with energy, while $h_{1}$ originates from a dimensionless coefficient that grows linearly with energy. Then, at high energies the eigenvalue difference is proportional to $E^{-1}$, just like a standard neutrino mass difference. More generally, using various combinations of masses and coefficients for Lorentz violation, similar models can be found that yield $E^{-1}, E^{-2}$, or $E^{-3}$ dependence at high energies, or $E^{1}, E^{2}$, or $E^{3}$ dependence at low energies. Further $E^{n}$ dependences can be obtained if the full 
$6 \times 6$ effective hamiltonian (4) is used.

\subsection{Bicycle model}

The complexity of the effective hamiltonian (4) and the range of possible neutrino behavior suggests that existing neutrino oscillation data may be compatible with an origin in Lorentz violation without mass differences. This is demonstrated explicitly by a simple model, called the bicycle model,${ }^{9}$ reproducing the major features of known neutrino behavior.

The bicycle model is a two-coefficient three-generation special case of the theory (4) without either mass-squared differences or $\nu \leftrightarrow \bar{\nu}$ mixing. It therefore involves only two degrees of freedom, rather than the four degrees of freedom used in the standard description with mass. The nonzero coefficients include an isotropic $c_{L}$ with nonzero element $\frac{4}{3}\left(c_{L}\right)_{e e}^{T T} \equiv 2 \stackrel{\circ}{c}>0$ and an anisotropic $a_{L}$ with degenerate nonzero real elements $\left(a_{L}\right)_{e \mu}^{Z}=$ $\left(a_{L}\right)_{e \tau}^{Z} \equiv \check{a} / \sqrt{2}$. These coefficients are understood to be specified in the standard Sun-centered frame $(T, X, Y, Z)$.

Diagonalizing the hamiltonian for the model yields ${ }^{9}$

$$
\begin{aligned}
P_{\nu_{e} \rightarrow \nu_{e}}= & 1-4 \sin ^{2} \theta \cos ^{2} \theta \sin ^{2}\left(\Delta_{31} L / 2\right) \\
P_{\nu_{e} \leftrightarrow \nu_{\mu}}= & P_{\nu_{e} \leftrightarrow \nu_{\tau}}=2 \sin ^{2} \theta \cos ^{2} \theta \sin ^{2}\left(\Delta_{31} L / 2\right) \\
P_{\nu_{\mu} \rightarrow \nu_{\mu}}= & P_{\nu_{\tau} \rightarrow \nu_{\tau}}=1-\sin ^{2} \theta \sin ^{2}\left(\Delta_{21} L / 2\right) \\
& \quad-\sin ^{2} \theta \cos ^{2} \theta \sin ^{2}\left(\Delta_{31} L / 2\right)-\cos ^{2} \theta \sin ^{2}\left(\Delta_{32} L / 2\right), \\
P_{\nu_{\mu} \leftrightarrow \nu_{\tau}}= & \sin ^{2} \theta \sin ^{2}\left(\Delta_{21} L / 2\right) \\
& \quad-\sin ^{2} \theta \cos ^{2} \theta \sin ^{2}\left(\Delta_{31} L / 2\right)+\cos ^{2} \theta \sin ^{2}\left(\Delta_{32} L / 2\right)
\end{aligned}
$$

where

$$
\begin{aligned}
& \Delta_{21}=\sqrt{(\stackrel{c}{c} E)^{2}+(\check{a} \cos \Theta)^{2}}+\stackrel{\circ}{c} E, \\
& \Delta_{31}=2 \sqrt{(\stackrel{\circ}{c} E)^{2}+(\check{a} \cos \Theta)^{2}}, \\
& \Delta_{32}=\sqrt{(\stackrel{c}{c} E)^{2}+(\check{a} \cos \Theta)^{2}}-\stackrel{\circ}{c} E, \\
& \sin ^{2} \theta=\frac{1}{2}\left[1-\stackrel{\circ}{c} E / \sqrt{(\stackrel{\circ}{c} E)^{2}+(\check{a} \cos \Theta)^{2}}\right],
\end{aligned}
$$

and where the propagation direction $\hat{p}$ is defined in Eq. (6). These probabilities also hold for antineutrinos.

Consider first the qualitative features of this theory. At low energies the coefficient $\check{a}$ induces oscillation of $\nu_{e}$ into an equal mixture of $\nu_{\mu}$ and $\nu_{\tau}$, while at high energies the coefficient $\stackrel{\AA}{c}$ controls the physics and there is no $\nu_{e}$ mixing. The critical energy for the theory is given by $E_{0}=|\check{a}| / \stackrel{\circ}{c}$. At energies $E \gg E_{0}$, Eq. (10) shows that $\sin ^{2} \theta$ effectively vanishes. The probabili- 
ties become those of a two-generation model with maximal $\nu_{\mu} \leftrightarrow \nu_{\tau}$ mixing and transition probability $P_{\nu_{\mu} \leftrightarrow \nu_{\tau}} \simeq \sin ^{2}\left(\Delta_{32} L / 2\right), \Delta_{32} \simeq \check{a}^{2} \cos ^{2} \Theta / 2 \stackrel{c}{c} E$. This theory therefore incorporates a Lorentz-violating seesaw yielding a pseudomass at high energies, having energy dependence like that of a conventional mass-squared difference $\Delta m_{\Theta}^{2}=\Delta m_{0^{\circ}}^{2} \cos ^{2} \Theta$, where $\Delta m_{0^{\circ}}^{2}=$ $\check{a}^{2} / \stackrel{c}{ }$. However, the value of the pseudomass $\Delta m_{\Theta}^{2}$ and therefore the behavior of neutrino oscillations vary with the declination $\Theta$ of the propagation. High-energy neutrinos propagating in the equatorial plane undergo no oscillation because all off-diagonal terms in $h_{\text {eff }}$ vanish, while those propagating parallel to celestial north or south behave according to the maximum pseudomass $\Delta m_{0^{\circ}}^{2}$.

The existing data for oscillations of atmospheric neutrinos ${ }^{31}$ are compatible with neutrino behavior in this model. To be specific, suppose $\Delta m_{0^{\circ}}^{2}=10^{-3} \mathrm{eV}^{2}$ and $E_{0}=0.1 \mathrm{GeV}$. This corresponds to $\stackrel{\circ}{c}=10^{-19}$, $\check{a}=10^{-20} \mathrm{GeV}$, values that are consistent with the notion of Planck-scale suppression. The value of $\Delta m_{0}^{2}$ lies near the accepted range adopted in the standard mass-based analysis, and $E_{0}$ then lies well below the energies relevant for atmospheric neutrino data. With these choices, high-energy atmospheric neutrinos exhibit the standard energy dependence even though they have zero mass differences, thanks to the Lorentz-violating seesaw. Moreover, within existing experimental resolution the zenith-angle dependence of the probability $P_{\nu_{\mu} \rightarrow \nu_{\mu}}$ averaged over the azimuthal angle is also similar to the standard maximal-mixing case with two generations and a mass-squared difference $\Delta m^{2}=2 \times 10^{-3} \mathrm{eV}^{2}$. Nonetheless, atmospheric-neutrino signals for Lorentz violation exist that are distinct from standard behaviors. For example, the theory predicts compass asymmetries, including significant azimuthal dependences. This can be seen by considering neutrinos propagating in the horizontal plane of the detector. Neutrinos coming from the north or south experience a pseudomass of $\Delta m_{\Theta}^{2}=\Delta m_{0^{\circ}}^{2} \cos ^{2} \chi$ and hence oscillate, while those coming from the east or west have $\cos \Theta=0$, $\Delta m_{\Theta}^{2}=0$ and hence experience no oscillations.

The basic solar-neutrino behavior predicted by the bicycle model is also consistent with observational data. ${ }^{31}$ Since the Earth's orbital plane lies at $\eta \simeq 23^{\circ}$ relative to the equatorial plane, and since solar neutrinos observed at the Earth are those propagating in the orbital plane, the value of $\cos ^{2} \Theta$ varies during the year. It is zero at the two equinoxes, and it reaches a maximum of $\sin ^{2} 23^{\circ}$ at the two solstices. Under the simplifying assumption of adiabatic propagation in the Sun, the average $\nu_{e}$ survival probability is $\left\langle P_{\nu_{e} \rightarrow \nu_{e}}\right\rangle_{\text {adiabatic }}=\sin ^{2} \theta \sin ^{2} \theta_{0}+\cos ^{2} \theta \cos ^{2} \theta_{0}$. Here, $\theta_{0}$ is the solar- 
core mixing angle, determined from Eq. (10) via substitution of $-\stackrel{i}{\mathrm{E}} \mathrm{\text {with }}$ $-\stackrel{c}{c} E+G_{F} n_{e} / \sqrt{2}$. It therefore follows that the predicted neutrino flux in the bicycle model is half the expected value at low energies and decreases at higher energies. This is consistent with existing data. Note that care is needed in interpreting the details of the time-dependent behavior. Although the adiabatic probability lies near the average during most of the year, the adiabatic approximation gives a strong suppression near the equinoxes. However, the model predicts that in fact oscillations cease at these times, so the adiabatic approximation fails then and the actual predicted survival probability spikes instead. The net combination of these effects near the equinoxes generates fluctuations in the binned flux, which offer a potential signal for Lorentz violation that could be sought in a detailed experimental analysis. The reader is cautioned, however, that although detection of the semiannual fluctuations represent a positive signal for Lorentz violation, the lack of such a signal is insufficient to exclude the bicycle model because simple changes to the model can yield only small semiannual fluctuations while maintaining the other neutrino behaviors discussed above. ${ }^{9}$

The predictions of the bicycle model are also consistent with neutrinooscillation data from other experiments. ${ }^{31}$ For instance, $\bar{\nu}_{e}$ survival is determined by the oscillation length $2 \pi / \Delta_{31}$, which is sufficiently small to affect KamLAND. ${ }^{41}$ The average $\bar{\nu}_{e}$ survival probability is $\left\langle P_{\bar{\nu}_{e} \rightarrow \bar{\nu}_{e}}\right\rangle=$ $1-2 \sin ^{2} \theta \cos ^{2} \theta \geq 1 / 2$, so the observed flux is predicted to be somewhat above half the flux expected in the absence of oscillations, as indeed reflected by the existing data. Similarly, tests of the bicycle model are also possible in long-baseline accelerator-based experiments, which involve $\nu_{\mu}$ oscillations at $\mathrm{GeV}$ energies and baselines of hundreds of kilometers. In particular, sidereal variations in $\nu_{\mu} \leftrightarrow \nu_{\tau}$ mixing are to be expected. The model also predicts that the results will vary with the beamline-direction of the experiment because the propagation angle $\Theta$ and hence the pseudomass $\Delta m_{\Theta}^{2}=\Delta m_{0^{\circ}}^{2} \cos ^{2} \Theta$ differ.

In summary, the bicycle model demonstrates that it is difficult and probably impossible at present to exclude the idea that observed neutrino oscillations originate from Lorentz and CPT violation rather than from mass differences. Positive and definitive signals for Lorentz and CPT violation arising from Planck-scale physics might be first revealed by detailed analysis of existing or near-future experimental data.

This work was supported in part by the United States Department of Energy under grant number DE-FG02-91ER40661 and by the National Aeronautics and Space Administration under grant number NAG8-1770. 


\section{References}

1. For recent overviews, see, for example, V.A. Kostelecký, ed., CPT and Lorentz Symmetry II, World Scientific, Singapore, 2002.

2. D. Colladay and V.A. Kostelecký, Phys. Rev. D 55, 6760 (1997); Phys. Rev. D 58, 116002 (1998).

3. V.A. Kostelecký, Phys. Rev. D, in press (hep-th/0312310).

4. S. Coleman and S. L. Glashow, Phys. Rev. D 59, 116008 (1999).

5. V. Barger et al., Phys. Rev. Lett. 85, 5055 (2000).

6. J.N. Bahcall, V. Barger, and D. Marfatia, Phys. Lett. B 534, 114 (2002).

7. A. de Gouvêa, Phys. Rev. D 66, 076005 (2002); G.L. Fogli et al., Phys. Rev. D 60, 053006 (1999); P. Lipari and M. Lusignoli, Phys. Rev. D 60, 013003 (1999); R. Foot et al., Phys. Lett. B 443, 185 (1998).

8. I. Mocioiu and M. Pospelov, Phys. Lett. B 537, 114 (2002).

9. V.A. Kostelecký and M. Mewes, hep-ph/0308300.

10. V.A. Kostelecký and M. Mewes, Phys. Rev. D 69, 016005 (2004).

11. S. Choubey and S.F. King, hep-ph/0311326.

12. V.A. Kostelecký and S. Samuel, Phys. Rev. D 39, 683 (1989); Phys. Rev. D 40, 1886 (1989); Phys. Rev. Lett. 63, 224 (1989); Phys. Rev. Lett. 66, 1811 (1991).

13. V.A. Kostelecký and R. Potting, Nucl. Phys. B 359, 545 (1991); Phys. Lett. B 381, 89 (1996); Phys. Rev. D 63, 046007 (2001); V.A. Kostelecký, M. Perry, and R. Potting, Phys. Rev. Lett. 84, 4541 (2000).

14. S.M. Carroll et al., Phys. Rev. Lett. 87, 141601 (2001); Z. Guralnik et al., Phys. Lett. B 517, 450 (2001); C.E. Carlson et al., Phys. Lett. B 518, 201 (2001); A. Anisimov et al., Phys. Rev. D 65, 085032 (2002); I. Mocioiu et al., Phys. Rev. D 65, 107702 (2002); M. Chaichian et al., hep-th/0212259.

15. R. Gambini and J. Pullin, in Ref. 1; J. Alfaro et al., Phys. Rev. D66, 124006 (2002); D. Sudarsky et al., Phys. Rev. Lett. 89, 231301 (2002); Phys. Rev. D 68, 024010 (2003); G. Amelino-Camelia, Mod. Phys. Lett. A 17, 899 (2002); Y.J. Ng, Mod. Phys. Lett. A18, 1073 (2003); R. Myers and M. Pospelov, Phys. Rev. Lett. 90, 211601 (2003); N.E. Mavromatos, hep-ph/0402005.

16. C.D. Froggatt and H.B. Nielsen, hep-ph/0211106.

17. J.D. Bjorken, Phys. Rev. D 67, 043508 (2003).

18. KTeV Collab., H. Nguyen, in Ref. 1; OPAL Collab., R. Ackerstaff et al., Z. Phys. C 76, 401 (1997); BELLE Collab., K. Abe et al., Phys. Rev. Lett. 86, 3228 (2001); BaBar Collab., B. Aubert et al., hep-ex/0303043; FOCUS Collab., J.M. Link et al., Phys. Lett. B 556, 7 (2003).

19. V.A. Kostelecký and R. Potting, Phys. Rev. D 51, 3923 (1995).

20. D. Colladay and V.A. Kostelecký, Phys. Lett. B 344, 259 (1995); Phys. Rev. D 52, 6224 (1995); Phys. Lett. B 511, 209 (2001); V.A. Kostelecký and R. Van Kooten, Phys. Rev. D 54, 5585 (1996); O. Bertolami et al., Phys. Lett. B 395, 178 (1997); V.A. Kostelecký, Phys. Rev. Lett. 80, 1818 (1998); Phys. Rev. D 61, 016002 (2000); Phys. Rev. D 64, 076001 (2001); N. Isgur et al., Phys. Lett. B 515, 333 (2001).

21. L.R. Hunter et al., in V.A. Kostelecký, ed., CPT and Lorentz Symmetry, 
World Scientific, Singapore, 1999; D. Bear et al., Phys. Rev. Lett. 85, 5038 (2000); D.F. Phillips et al., Phys. Rev. D 63, 111101 (2001); M.A. Humphrey et al., Phys. Rev. A 62, 063405 (2000); Phys. Rev. A 68, 063807 (2003); V.A. Kostelecký and C.D. Lane, Phys. Rev. D 60, 116010 (1999); J. Math. Phys. 40, 6245 (1999).

22. R. Bluhm et al., Phys. Rev. Lett. 88, 090801 (2002); Phys. Rev. D 68, 125008 (2003).

23. F. Canè et al., physics/0309070.

24. H. Dehmelt et al., Phys. Rev. Lett. 83, 4694 (1999); R. Mittleman et al., Phys. Rev. Lett. 83, 2116 (1999); G. Gabrielse et al., Phys. Rev. Lett. 82, 3198 (1999); R. Bluhm et al., Phys. Rev. Lett. 82, 2254 (1999); Phys. Rev. Lett. 79, 1432 (1997); Phys. Rev. D 57, 3932 (1998).

25. B. Heckel, in Ref. 1; L.-S. Hou et al., Phys. Rev. Lett. 90, 201101 (2003); R. Bluhm and V.A. Kostelecký, Phys. Rev. Lett. 84, 1381 (2000).

26. S.M. Carroll et al., Phys. Rev. D 41, 1231 (1990); V.A. Kostelecký and M. Mewes, Phys. Rev. Lett. 87, 251304 (2001).

27. R. Jackiw and V.A. Kostelecký, Phys. Rev. Lett. 82, 3572 (1999); C. Adam and F.R. Klinkhamer, Nucl. Phys. B 657, 214 (2003); H. Müller et al., Phys. Rev. D 67, 056006 (2003); T. Jacobson et al., Phys. Rev. D 67, 124011 (2003); V.A. Kostelecký et al., Phys. Rev. D 65, 056006 (2002); Phys. Rev. D 68, 123511 (2003); V.A. Kostelecký and A.G.M. Pickering, Phys. Rev. Lett. 91, 031801 (2003); R. Lehnert, Phys. Rev. D 68, 085003 (2003); G.M. Shore, gr-qc/0304059.

28. J. Lipa et al., Phys. Rev. Lett. 90, 060403 (2003); H. Müller et al., Phys. Rev. Lett. 91, 020401 (2003).

29. V.A. Kostelecký and M. Mewes, Phys. Rev. D 66, 056005 (2002).

30. V.W. Hughes et al., Phys. Rev. Lett. 87, 111804 (2001); R. Bluhm et al., Phys. Rev. Lett. 84, 1098 (2000).

31. The available data are summarized in The Particle Data Group, K. Hagiwara et al., Phys. Rev. D 66, 010001 (2002) (see also http://pdg.lbl.gov).

32. V.A. Kostelecký and R. Lehnert, Phys. Rev. D 63, 065008 (2001).

33. M. Gell-Mann et al., in P. van Nieuwenhuizen and D.Z. Freedman, ed., Supergravity, North Holland, Amsterdam, 1979; T. Yanagida, in O. Sawada and A. Sugamoto, eds., Workshop on Unified Theory and the Baryon Number of the Universe, KEK, Japan, 1979; R. Mohapatra and G. Senjanović, Phys. Rev. Lett. 44, 912 (1980).

34. D. Colladay and P. McDonald, J. Math. Phys. 43, 3554 (2002).

35. M.S. Berger and V.A. Kostelecký, Phys. Rev. D 65, 091701(R) (2002).

36. G. Barenboim and J. Lykken, Phys. Lett. B 554, 73 (2003).

37. O.W. Greenberg, Phys. Rev. Lett. 89, 231602 (2002); Phys. Lett. B 567, 179 (2003).

38. R. Brustein, D. Eichler, and S. Foffa, Phys. Rev. D 65, 105006 (2002).

39. L. Wolfenstein, Phys. Rev. D 17, 2369 (1978); S. Mikheev and A. Smirnov, Sov. J. Nucl. Phys. 42, 913 (1986).

40. LSND Collab., C. Athanassopoulos et al., Phys. Rev. Lett. 81, 1774 (1998).

41. KamLAND Collab., K. Eguchi et al., Phys. Rev. Lett. 90, 021802 (2003). 